\title{
MALIGNANT MELANOMA OF THE LACRIMAL SAC*
}

\author{
BY \\ I. M. DUGUID \\ Department of Pathology, Institute of Ophthalmology, University of London
}

REPORTS on tumours of the lacrimal sac appear infrequently in the literature and most ophthalmologists seldom encounter these tumours, but this does not detract from their interest, occasional reports serving as a reminder of the possible presence of a neoplasm when dealing with a mass in this region.

Since 1950, eight carcinomata, one adenoma, three papillomata, and two malignant melanomata have been encountered in this department. These figures, as far as melanomata are concerned, give a somewhat erroneous impression of their comparative incidence, for only three malignant melanomata of the lacrimal sac have so far been reported in the world literature-one each from Russia, Italy, and France (Muravleškin, 1926; Margotta, 1929; Florentin, Thomas, and Poirot, 1947). Interestingly, all three tumours were found in females and were situated on the left side.

Early diagnosis of malignancy is of great importance with regard to the patient's expectancy of life, and for this reason alone it would seem advantageous to study more closely malignant melanomata of the sac about which there is a paucity of detail in the literature.

\section{Case Reports}

Case 1, a woman aged 57 years, attended hospital in June, 1961, with a blood-stained discharge from the left eye and a painless swelling of 2 months' duration in the region of the left lacrimal sac.

Examination.-The clinical features resembled those of a mucocoele but pressure on the overlying skin failed to reduce the mass then thought to be lacrimal sac filled by inspissated pus. Small but persistent haemorrhages from the left punctum followed lacrimal syringing which also revealed freely patent passages. During the 2 succeeding months, syringing was repeated and each time patency of the passages was noted but haemorrhage followed. The mass itself did not appreciably alter in size. 3 months after the first attendance, pressure on the skin overlying the sac expressed a lot of blood clot both upwards through the punctum and downwards into the inferior meatus of the nose. Gradually, as the mass became harder, it was suspected that the lesion might well be neoplastic in nature rather than a mucocoele, so it was decided to explore the sac with the idea of possibly going on to do a dacryocystorhinostomy.

Operation.-5 months after the first attendance at hospital, the lacrimal sac was exposed; it was found to be hardened, and obviously infiltrated by a slightly darkened tumour which could only be removed piecemeal; two small fragments were sent to the Department of Pathology at the Institute of Ophthalmology.

Histopathology.-The appearances of the two fragments were similar and showed fibrosis of the wall of the lacrimal sac, scattered chronic inflammatory cells, and a nodule of irregular, densely

* Received for publication November 11, 1963. 
cellular tumour besides some haemorrhage. The lumen of the sac contained not only haemorrhage but also some cellular debris, consisting of intermingled chronic inflammatory cells, desquamated epithelium, and tumour cells, similar to those in the wall of the sac (Fig. 1).

FIG. 1.-Low-power view, showing lumen of sac (A) containing cellular detritus and haemorrhage, and lacrimal epithelium (B) which has been partially infiltrated by round cells and neoplastic cells. $\times 90$.

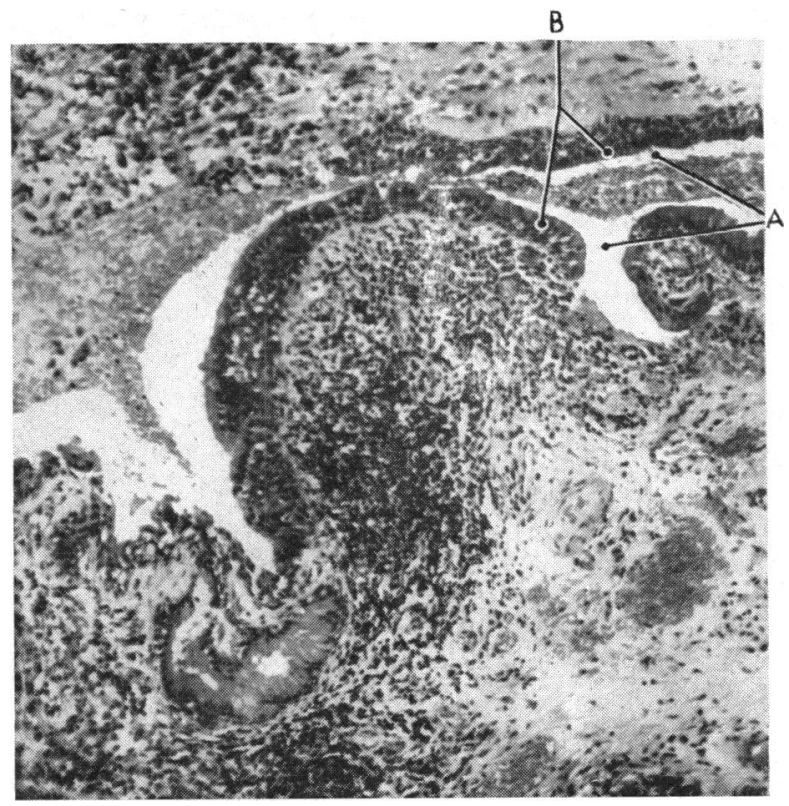

An irregular tumour mass, which had completely replaced the wall of the sac in places, was composed of masses of anaplastic spindle and epithelioid cells (Fig. 2), with some scattered haemorrhage and abundant melanin (Fig. 3) which was removed by bleaching.

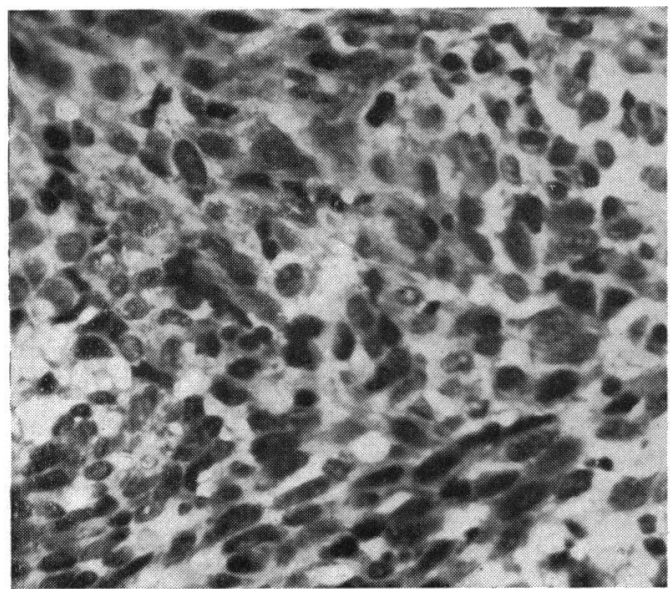

FIG. 2.-Higher-power view showing cytology of malignant melanoma. $\times 390$.

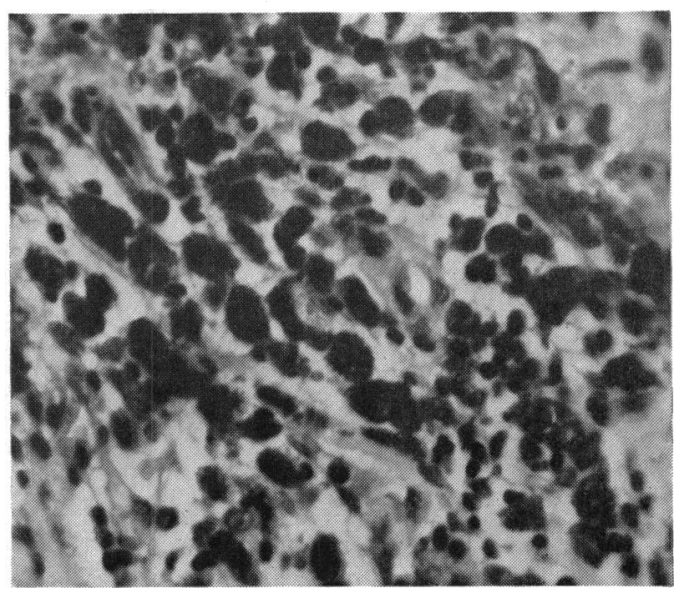

FIG. 3.-Another portion of the tumour containing abundant melanin. $\times 390$.

Mitoses were numerous and the cytology was of malignant type. In one area the epithelium of the sac had not only been invaded but had also been replaced by tumour cells. There was no evidence to suggest that this neoplasm arose from a conjunctival intra-epithelial melanoma (precancerous melanosis).

Once the diagnosis of malignant melanoma of the lacrimal sac was established, the patient was given a course of super-voltage irradiation to the left orbital region.

Follow-up.- It is satisfactory to note that the patient has been fit for a year since her operation. 
Case 2, a man aged 70 years, had been attending hospital for 2 years because of failing vision due to cataract before he complained of excessive watering from the right eye in March, 1963.

Examination.-Lacrimal syringing via the upper punctum and canaliculus, revealed freely patent passages. About 2 months later, he first experienced haemorrhage from the lacrimal punctum and on the following morning, a cystic mass in the region of the right sac could be reduced by digital pressure on the overlying skin, only to be quickly followed by epistaxis. As the lesion was likely to be neoplastic in origin, dacryocystectomy was considered desirable, but unfortunately the patient then refused surgery.

During the succeeding 3 days, however, intermittent haemorrhage became more persistent and the patient finally consented to surgery.

Operation.-The sac was excised but the underlying bone was found to be eroded. About a month later, a nodular growth was found pushing up the operation scar and since this was believed to be a recurrence of the original neoplasm the patient was referred for radiotherapy.

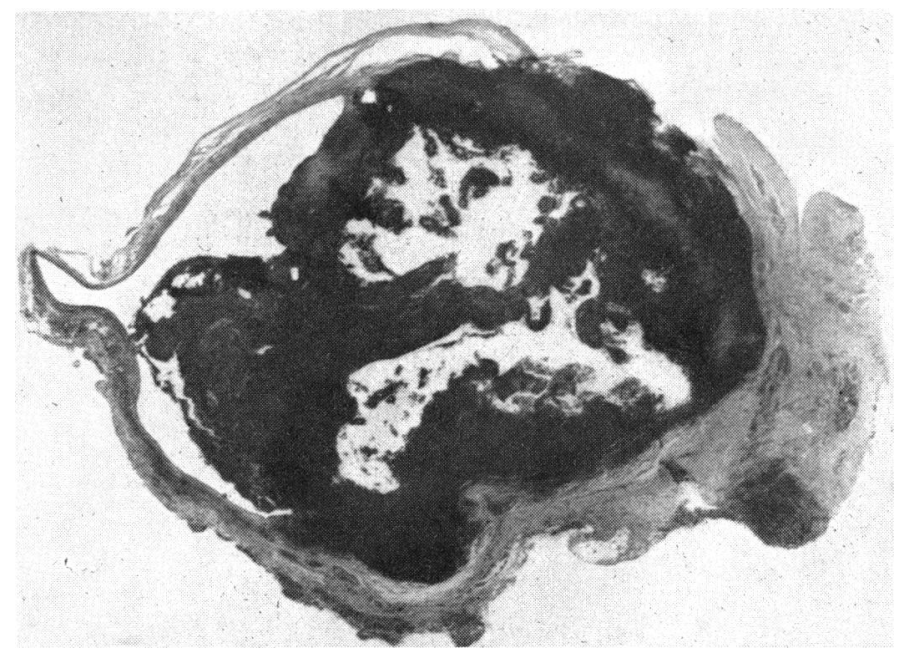

FIG. 4.-Low-power view showing transverse section of sac heavily infiltrated by malignant melanoma. $\times 8$.

Histopathology.-The wall of the lacrimal sac (Fig. 4) was extensively replaced by a large, highly malignant tumour consisting of hyperchromatic polyhedral cells which themselves showed numerous mitoses and were heavily pigmented in one area (Fig. 5, opposite).

Many thin-walled blood spaces and chronic inflammatory cells were scattered throughout the tumour mass, while haemorrhage and cellular detritus were present in the lumen of the sac. Most of the epithelium of the sac had been replaced by this neoplasm which histologically was a malignant melanoma of the lacrimal sac (Fig. 6, opposite).

\section{Discussion}

Malignant melanomata of the lacrimal sac would appear to have several clinical features in common with other tumours arising in this region; in fact, the similarity is often so marked that their initial differentiation is impossible. The patient may present at hospital with a watering eye with or without any palpable tumour in the region of the sac or may attend with signs and symptoms resembling those of a simple dacryocystitis. Epiphora appears to be a constant complaint and syringing usually demonstrates patency of the lacrimal passages. Initially, this epiphora in the presence of patent passages may be puzzling, but it is readily understandable when 

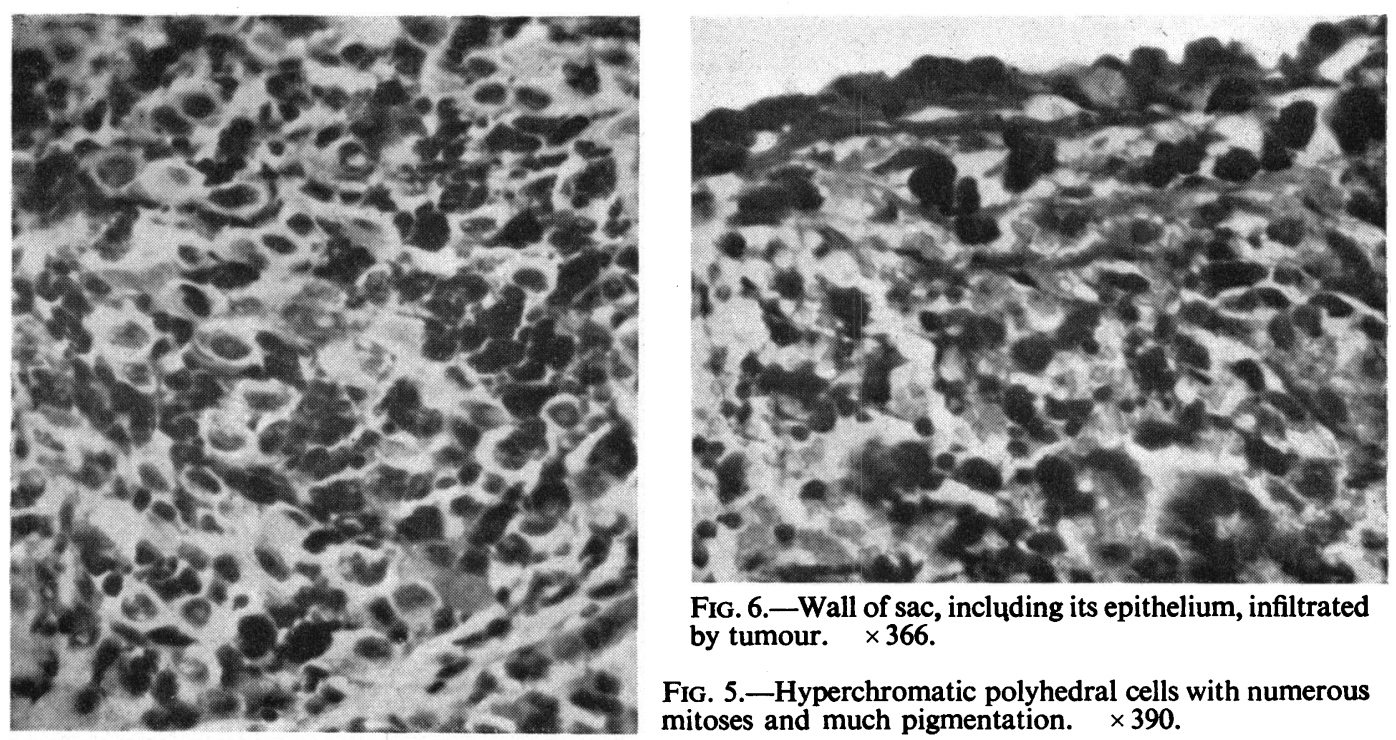

Fig. 6. - Wall of sac, including its epithelium, infiltrated by tumour. $\times 366$.

Fig. 5.-Hyperchromatic polyhedral cells with numerous mitoses and much pigmentation. $\times 390$.

one considers that the sac wall is probably inelastic, being already infiltrated by tumour cells, which have not as yet appreciably encroached upon the sac lumen. Accompanying this neoplastic invasion of the wall is a chronic inflammatory infiltration, and features of a dacryocystitis may be superimposed upon a hitherto ostensibly simple or uncomplicated epiphora, if the inflammatory element has not already been present or predominant. This apparent dacryocystitis in the presence of patent passages may even continue over a long period and is unresponsive to antibiotics, but eventually tumour formation is evident and the onset of haemorrhage into the tear passages may occur spontaneously or may follow lacrimal syringing. After the onset of haemorrhage the tumour mass is palpable but may not be entirely reducible. Both these features should make the clinician think of malignant change rather than of an uncomplicated dacryocystitis. With increasing infiltration by the tumour, haemorrhage may not only result from pressure on the mass but may also become more frequent and persistent.

The dark colour of the mass may suggest melanoma, but it should be remembered that haemorrhage into the other neoplasms may give a similar appearance although at this stage the diagnosis is often more obvious; moreover, metastasis to the preauricular, submaxillary, and cervical lymph nodes would probably have occurred.

Treatment consists of excision of the entire neoplastic mass together with postoperative irradiation to the area. Biopsy or incomplete excision is inadequate. Radiotherapy following surgery to malignant melanomata of the sac would seem advantageous, although an experience of only two cases is obviously insufficient to express an opinion of its value. The first of the two cases examined here had postoperative radiotherapy, but in the second case none was given for 1 to 2 months before the appearance of a nodular recurrence in the operation site. The second case reported here parallels that recorded by Florentin and others (1947) with regard to early recurrence. 
The exact origin of malignant melanomata of the lacrimal sac is uncertain but it seems likely that they arise from the melanocyte, a mature melanin-forming cell. In the skin, melanocytes are found between the epithelial cells along the dermoepidermal junction and, as the definitive lacrimal sac is eventually derived from an embryonic ectodermal downgrowth, it is therefore probable that melanocytes are also similarly situated between the epithelial cells of the sac. On the other hand, these lacrimal melanomata could arise from melanoblasts, or immature melaninforming cells, and it may be that a parallel is found in the true melanomata of the cornea for the basal cells of the cornea are potential melanoblasts.

Despite the speculation about their origin, early diagnosis of these neoplasms is essential - exact diagnosis may not be easy or possible in the early stages, but all the features indicating malignancy should be carefully and repeatedly sought. Once malignancy is suspected, surgery should be adequate and entail the removal of any extensions of the neoplasm along the naso-lacrimal canal, for both rapid increase in size and metastasis-follow only too readily incomplete removal.

\section{Summary}

(1) Two cases of malignant melanoma of the lacrimal sac are described in detail.

(2) The clinical features, diagnosis, and management of the condition are discussed. The need for diagnosis and treatment is emphasized.

I am particularly indebted to Prof. N. Ashton for his valuable assistance during the preparation of this paper, and to Mr. A. J. Boase, St. John Ophthalmic Hospital, Jerusalem, for kindly supplying the clinical data from Case 2. In addition, I wish to thank Mr. V. E. Elwood for technical assistance and Miss C. von Mutius for secretarial help.

\section{REFERENCES}

Florentin, Thomas, C., and Poirot, J. C. (1947). Bull. Soc. Ophtal. Paris, No. 2., p. 270.

Margotta, G. (1929). Ann. Ottal., 57, 387.

MURAVLEŠKin, N. (1926). Russ. oftal. Ž., 5, 897. 